\title{
PERFORMANCE ASSESSMENT DURING MILITARY AIRCRAFT OPERATIONAL VIBRATION EXPOSURE
}

\author{
*Suzanne D. Smith, PhD ${ }^{1}$, Jennifer G. Jurcsisn ${ }^{1,}$ Cecelia J. Harrison ${ }^{2}$ \\ ${ }^{1}$ Air Force Research laboratory, Wright-Patterson AFB OH \\ ${ }^{2}$ Infoscitex Corporation, Dayton, $\mathrm{OH}$
}

\section{Introduction}

Military aircrew flying long duration missions aboard rotary-wing and fixed-wing propeller aircraft have repeatedly reported anecdotal symptoms of lower extremity numbing, discomfort, and musculoskeletal pain. Exposure to higher frequency vibration has been cited as a potential contributor. Vibration surveys of these aircraft ${ }^{1}$ have shown that the acceleration levels may cause discomfort with the potential for health risk during prolonged and repeated exposures. ${ }^{2}$ It is expected that these levels could also affect aircrew performance during long duration missions. Limited studies have focused on the cognitive effects of vibration. Performance studies conducted using lower frequency vibration $(<10 \mathrm{~Hz})$ may have introduced errors due to motion disturbances in manual and visual control. One study suggested that monotonous low frequency vibration $(<10 \mathrm{~Hz})$ has a tiring effect. ${ }^{3}$ Exposure to $16 \mathrm{~Hz}$ sinusoidal vibration did show impairments in short term memory. Mean reaction time and attentional lapses were degraded during exposures to $1.0,1.6$, and $2.5 \mathrm{~ms}^{-2} \mathrm{rms}$. Interestingly, response errors were significantly higher at $1.0 \mathrm{~ms} \mathrm{rms}^{-2}$ only, suggesting compensatory activity. ${ }^{4}$ This study investigated the feasibility of producing changes in performance during exposure to operational vibration. Task performance, workload perception, and subjective vibration and discomfort assessments were used to evaluate the effects of exposure type and duration.

\section{Methods}

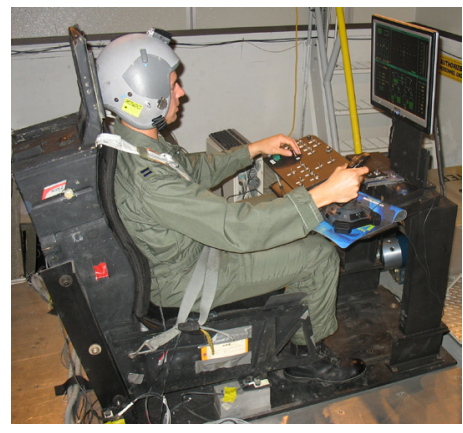

Figure 1. Subject Performing MAT-B Tasks.

Eight male and female subjects were exposed to operational vibration associated with the weather officer station in the WC-130J Hercules and the pilot station in the CV-22 Osprey. Four subjects also participated in the No Vibration condition (NO VIBE). The NASA MultiAttribute Task Battery (MAT-B) equipped with a joystick, toggle switches, and display were used to measure performance (communication response time and error, dials response time and error, lights response time and error, and tracking error) during three consecutive 30-minute exposure intervals (Fig. 1). The NASA Task Load Index (TLX) was used to assess subject workload perception for each interval based on six factors (mental demand, physical demand, temporal demand, own performance, effort, and frustration). The Vibration and Comfort Survey was used to assess subject perception of the vibration and discomfort at the face, head/neck, chest, upper back, lower back, buttocks, upper legs, lower legs, and feet for each interval via a numerical rating system. Vibration sessions were repeated three times; only one NO VIBE session was conducted. 


\section{Results}

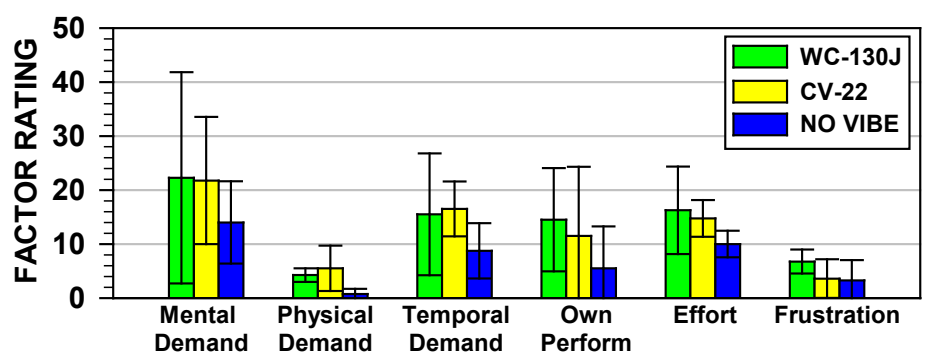

Figure 2. Workload Factor Ratings

(4 Subjects, Vibe Test 3, 90 Min)

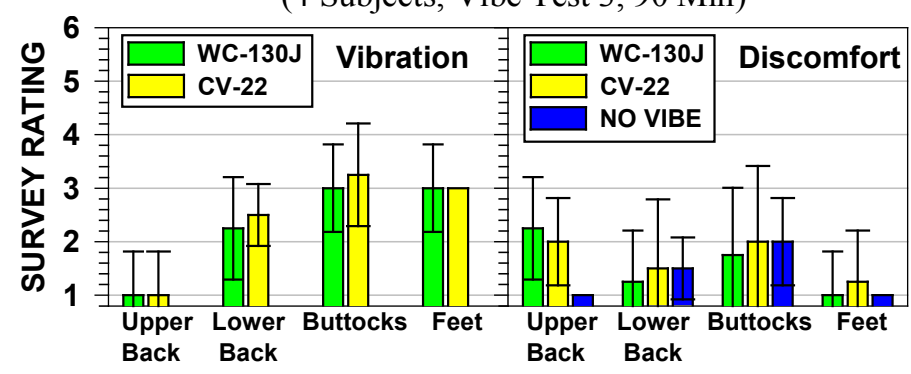

Figure 3. Vibration and Discomfort Survey Ratings

(4 Subjects, Vibe Test 3, 90 Min)

The highest weighted seat pan acceleration occurred for the CV22 in the vertical direction $(\sim 16$ $\mathrm{Hz}$ ) at levels associated with being fairly uncomfortable. ${ }^{1}$ In general, there were no significant effects of exposure type and duration on task performance (Repeated Measures ANOVA and Bonferroni

Comparison Test). The highest and most variable response time occurred with the dials task. No clear trends were observed in task variability over the 30 -minute intervals. In general, there were no significant effects of exposure type and duration on the workload ratings. The lowest workload was associated with physical demand and frustration (Fig. 2). The vibration was felt more in the lower torso (Fig. 3). Higher discomfort was observed for the upper back, lower back, and buttocks (Fig. 3) with most ratings less than 3. The test conductors observed notable subject fatigue (sleepiness) by the end of the exposures. The subjects indicated that the NO VIBE condition made them less sleepy than either vibration condition.

\section{Discussion}

The higher frequency operational vibration acceleration levels appeared to have no influence on task performance, workload perception, and vibration/discomfort assessment, although observations do suggest an effect on sleepiness. Discomfort was not necessarily associated with feeling the vibration; prolonged static posture may play a role. The MAT-B tasks did not appear to be sensitive to the fatigue observed during the study. It was not clear whether task performance was affected by compensatory activity. A follow-on investigation will evaluate alternative tasks that specifically challenge vigilance, working and recognition memory, and decision-making. These types of tasks may influence the perception of workload and vibration/discomfort over time.

\section{References}

1. International Standards Organization (ISO). Mechanical vibration and shock-Evaluation of human exposure to whole-body vibration-Part 1: General requirements. ISO 2631-1: 1997.

2. Smith, S.D., Jurcsisn, J.G., and Bowden, D.R. (2008). CV-22 human vibration evaluation. AFRL-RHWP-TR-2008-0095. Air Force Research Laboratory, Wright-Patterson AFB OH USA.

3. Landstrom, U. and Lunstrom, R. (1985). Changes in wakefulness during exposure to whole-body vibration. Electroencephalography and Clin Neurophysiology. 61, 411-415.

4. Sherwood, N. and Griffin, M.J. (1990). Effects of whole-body vibration on short-term memory.

Aviation, Space, and Environmental Medicine. December, 1092-1097. 